\title{
Emprego de maravalhas de Eucalyptus na produção de painéis: uma alternativa para aproveitamento dos resíduos oriundos da usinagem da madeira
}

\author{
Douglas Lamounier Faria ${ }^{1 *}$ Thamirys Andrade Lopes ${ }^{2}$ Daiane Erika Lopes ${ }^{1}$ Bruno Cassiano Lemos \\ Araújo ${ }^{1}$ Gustavo Mesquita de Castro ${ }^{1}$ Lourival Marin Mendes ${ }^{1}$ José Benedito Guimarães Junior $^{1}$
}

${ }^{1}$ Universidade Federal de Lavras, R. Aquenta Sol, s/n- CEP 37200-900, Lavras, MG, Brasil

${ }^{2}$ Universidade Federal de Viçosa, Av. Peter Henry Rolfs, s/n - Campus Universitário, CEP 36570-900, Viçosa, MG, Brasil

Original Article
*Corresponding author:
douglas.lamounier@yahoo.c
om
Keywords:
Mechanical resistance
Dimensional stability
Engineered wood panels
Palavras-chave:
Resistência mecânica
Estabilidade dimensional
Painéis de madeira
reconstituída
Received in
2020/02/28
Accepted on
2020/06/26
Published in
2020/11/02
(cc) B.34062/afs.v7i3.9895
B

RESUMO: O objetivo deste trabalho foi avaliar as propriedades físicas e mecânicas de chapas de madeira aglomerada utilizando partículas oriundas de maravalhas a três teores de adesivo fenol-formaldeído, 6, 9 e 12\% (base massa seca). Os painéis foram produzidos com densidade nominal de 0,70 g.cm-3, ciclo de prensagem com temperatura de $160{ }^{\circ} \mathrm{C}$, pressão de $3,92 \mathrm{MPa}$ e tempo de 8 minutos. Foram avaliadas as propriedades de absorção de água (AA), inchamento em espessura (IE), ambas após 2 e 24 horas, densidade aparente, razão de compactação, tração perpendicular, módulo de elasticidade (MOE) e módulo de ruptura (MOR) na flexão estática e resistência ao arranchamento de parafuso. Os resultados dos testes indicaram que com o aumento do teor de adesivo de 6 para $12 \%$, não houve diferença estatística significativa para os valores médios de densidade aparente e razão de compactação, sendo eles 0,624 g.cm-3 e 1,234, respectivamente, e diminui-se a AA e o IE após 2 e 24 horas. Houve um ganho de $49 \%$ para MOR e $40 \%$ para MOE. Para tração perpendicular, os maiores valores médios foram referentes ao painel com $12 \%$ de adesivo. Já para arrancamento de parafuso, os painéis não atenderam às normas vigentes.

\section{Use of Eucalyptus wood shavings in the production of panels: an alternative for the use of residues from wood machining}

\begin{abstract}
The aim of this paper is to evaluate the physical and mechanical properties of particleboards using particles originated from wood shavings with three levels of phenol-formaldehyde adhesive, 6, 9 and 12\% (dry mass basis). The panels were produced with nominal density of $0.70 \mathrm{~g} . \mathrm{cm}-3$, pressing cycle with temperature of $160{ }^{\circ} \mathrm{C}$, pressure of $3.92 \mathrm{MPa}$ and time of 8 minutes. The properties of water absorption (AA), swelling in thickness (IE), both after 2 and 24 hours, bulk density, compression ratio, perpendicular traction, modulus of elasticity (MOE) and modulus of rupture (MOR) in static bending and resistance to screw withdrawal were evaluated. The test results indicated that with the increase of the adhesive content from 6 to $12 \%$, there was no statistically significant difference for the mean values of bulk density and compression ratio, being $0.624 \mathrm{~g} . \mathrm{cm}-3$ and 1.234, respectively, and the AA and IE decreased after 2 and 24 hours. There was a $49 \%$ gain for MOR and $40 \%$ for MOE. For perpendicular traction, the highest mean values were for the panel with $12 \%$ adhesive. As for screw withdrawal, the panels did not meet current standards.
\end{abstract}




\section{Introdução}

A cadeia produtiva da madeira gera uma grande quantidade de resíduos sólidos em todas as suas etapas produtivas, desde o plantio até as consecutivas transformações que dão origem ao produto final. Devido ao alto volume de resíduos, faz-se necessário um plano de gestão dos mesmos para que esse material possa ser transformado e reinserido no ciclo econômico da madeira (Silva 2018).

Os resíduos produzidos no processamento da madeira podem ser a serragem ou pó de serra, costaneiras, resíduos do refilamento, maravalhas, aparas ou destopo e casca (Fagundes 2003). As maravalhas são resíduos resultantes das etapas de desengrosso e aplainamento das tábuas e possui um formato característico e de fácil identificação.

As empresas do setor de florestas plantadas adotam como prática a gestão rigorosa dos resíduos sólidos de suas atividades, visando reduzir a geração, assegurar a destinação correta e atender aos requisitos legais e de certificação relativos ao assunto. Em 2018, o setor gerou 52 milhões de toneladas de resíduos sólidos, sendo que, desse total, 36,9 milhões $(70,9 \%)$ foram gerados pelas atividades florestais e 15,1 milhões $(29,1 \%)$ pelas industriais (Ibá 2019).

Uma das razões para esse alto percentual de rejeitos é a falta de conhecimento do processamento adequado das espécies florestais (Melo et al. 2012) uso de maquinários e equipamentos obsoletos, layout inadequado e precário das serrarias e de novas tecnologias que permitam um maior aproveitamento da madeira (Cerqueira et al. 2012).

Esses resíduos são estocados em determinado local na serraria para serem biologicamente degradados, além de ocuparem um espaço físico que poderia ser melhor utilizado, são queimados sem nenhum fim energético ou atirados nos rios resultando em impactos ambientais negativos e perdas econômicas significativas (Silva et al. 2017).

Apesar de a legislação apontar a auto responsabilidade das empresas na remoção, estocagem e tratamento de resíduos gerados pelos processos produtivos (Brasil 2010) na cadeia moveleira de regiões mais afastadas, é comum o resíduo gerado na produção de móveis ser destinado de forma incorreta ou vendido a um preço muito baixo para padarias e olarias regionais, resultando em impactos ambientais negativos e perdas econômicas significativas (Amaral et al. 2018).

A tecnologia da madeira é de suma importância para auxiliar no aproveitamento de resíduos e melhorar a taxa de conversão das toras (Merry et al. 2009).

No entanto é necessário o conhecimento das propriedades físicas das espécies, para definir o uso, desenvolvimento e geração de novos produtos utilizando os resíduos madeireiros. $\mathrm{O}$ reaproveitamento desses resíduos para a fabricação de chapas de madeira aglomerada pode se tornar uma alternativa na tentativa de mitigar impactos ambientais, além de agregar valor ao mesmo, passando a ser uma nova fonte de matéria-prima.

Existem alguns trabalhos na literatura que empregam resíduos, dentre eles as maravalhas, para a produção de painéis de madeira. Iwakiri et al. (2012) avaliaram a qualidade de painéis aglomerados produzidos com resíduos de processamento em serraria de nove espécies de madeiras tropicais da Amazônia. Os resultados dos testes indicaram a viabilidade técnica de utilização de todas as espécies estudadas na produção de painéis de madeira aglomerada. Fiorelli et al. (2014) estudaram o potencial da fabricação de painéis de partículas com resíduo de madeira da espécie Pinus spp. e os resultados mostraram que os painéis atingiram as normas de comercialização requeridas, indicando que é possível o uso de resíduos para produção de aglomerados. Hazrati-Behnagh et al., (2015) avaliaram as propriedades térmicas, mecânicas e isolantes de painéis aglomerados de baixa densidade utilizando resíduos de bagaço de cana-de-açúcar e mix de maravalhas de várias espécies e concluíram que os painéis conseguiram reduzir o coeficiente de ruído do ambiente, estavam dentro da faixa de valores para isolamento térmico e apresentaram boas propriedades mecânicas, atendendo todas as normas das respectivas propriedades. Cabral et al. (2017) avaliaram a viabilidade de uso de fibras de sisal associada à maravalha de Pinus spp. como matérias-primas constituintes de painéis e os resultados obtidos indicaram a viabilidade de produção dos painéis aglomerados, em que aqueles produzidos com $25 \%$ de fibras e $75 \%$ de maravalhas apresentaram propriedades termo-físico-mecânicas que atenderam as prescrições mínimas estabelecidas nos documentos normativos para painéis de baixa densidade.

Em trabalhos citados anteriormente, as maravalhas passaram por processos de homogeneização das partículas através do uso de moinhos e peneiras classificatórias. Já Silva (2018), fez um estudo da viabilidade técnica do reaproveitamento da maravalha de forma integral, sem passar por nenhum processo de homogeneização, para a produção de painéis aglomerados e concluiu que os painéis produzidos têm potencial para serem utilizados como parte de um sistema de isolamento térmico em construções do tipo wood frame devidos aos resultados obtidos.

Diante do exposto, o objetivo deste trabalho foi avaliar as propriedades físicas e mecânicas de painéis de madeira aglomerada utilizando maravalhas oriundas do processo de desengrosso de tábuas, a três teores de adesivo fenol-formaldeído.

\section{Material e Métodos}




\section{Obtenção do material}

O experimento foi realizado na Unidade Experimental de Produção de Painéis de Madeira (UEPAM) na Universidade Federal de Lavras (UFLA). As maravalhas utilizadas para a produção dos painéis aglomerados foram aproveitadas do processamento da madeira na etapa de desengrosso das tábuas de Eucalyptus grandis. A madeira que gerou as maravalhas apresentaram densidade básica de $0,503 \pm 0,079$ g.cm-3. O adesivo utilizado foi o fenol-formaldeído, que apresentou teor de sólidos de $60 \%$, viscosidade de $200,95 \mathrm{cP}, \mathrm{pH}$ de 8,45 e gel timer de 7,85 min.

As partículas de maravalhas foram peneiradas em malhas com abertura de 9,5 a $16 \mathrm{~mm}$, com o objetivo de eliminar os finos e obter maravalhas com dimensões em torno de 12 × 8 × 1 $\mathrm{mm}$ (comprimento $\mathrm{x}$ largura $\mathrm{x}$ espessura). Posteriormente as partículas foram colocadas em estufa à temperatura de $70 \pm 2{ }^{\circ} \mathrm{C}$ até atingir teor de umidade de $5 \%$ (base massa seca) e em seguida, aferida a massa para a produção dos painéis.

\section{Confecção dos painéis aglomerados}

Os painéis foram produzidos com densidade nominal de 0,70 g.cm-3, sendo avaliados três diferentes tratamentos com variações de 6, 9 e $12 \%$ no percentual de adesivo fenol-formaldeído. Cada tratamento contou com três repetições, totalizando 9 painéis.

As maravalhas foram colocadas em um tambor giratório (Figura 1), separadamente, para aplicação do adesivo, através de pulverização. Em seguida foram colocadas em uma forma com as dimensões de $300 \mathrm{~mm}$ de largura por $300 \mathrm{~mm}$ de comprimento, sendo, em seguida, pré-prensadas, e finalmente prensadas. A espessura dos painéis foi de $1,50 \mathrm{~mm}$. A prensagem foi feita em uma prensa de pratos planos e horizontais com aquecimento elétrico, a $160{ }^{\circ} \mathrm{C}$ e 3,92 $\mathrm{MPa}$ de pressão, por um período de 8 minutos. Após a prensagem, os painéis foram acondicionados numa câmara climática à temperatura de $20 \pm 3^{\circ} \mathrm{C}$ e umidade relativa de $65 \pm$ $5 \%$ até atingir a umidade de equilíbrio aproximado de $12 \%$ e término do processo de solidificação do adesivo.
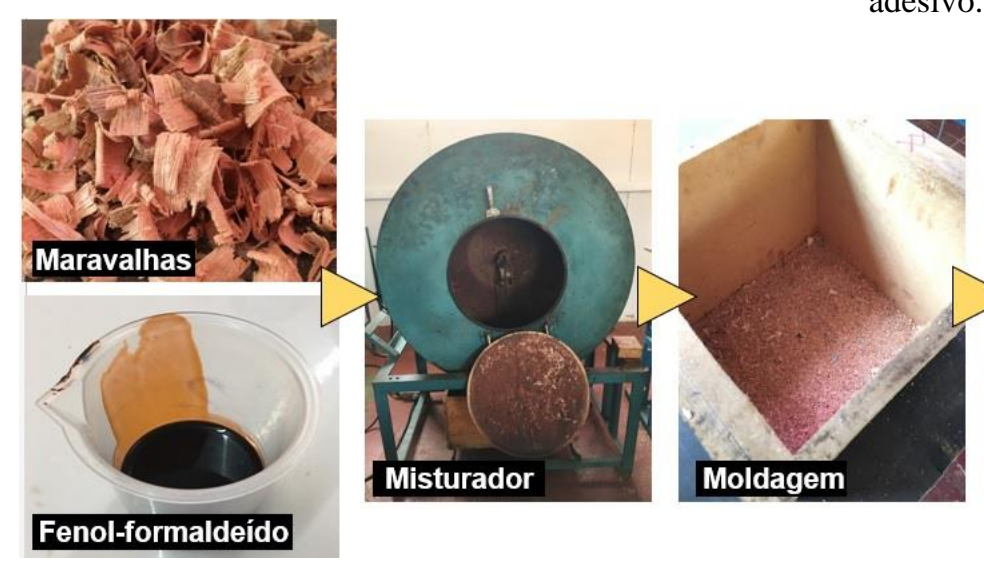
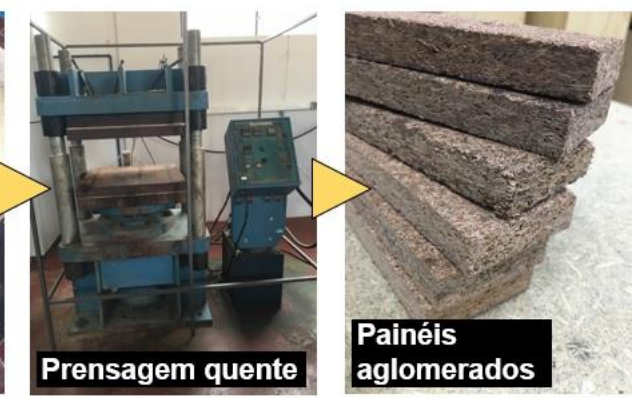

Figura 1. Processo de produção dos painéis aglomerados.

\section{Análise das propriedades físicas e mecânicas dos painéis aglomerados}

A razão de compactação de cada painel aglomerado foi calculada através da razão da densidade aparente do painel pela densidade básica da madeira de Eucalyptus grandis.

Para a avaliação das propriedades de absorção de água após 2 e 24 h de imersão (AA2h e AA24h) e inchamento em espessura após 2 e 24 h (IE2h e IE24h) de imersão foi empregada a norma D-1037 (ASTM 2012), utilizando 10 corpos de prova para cada tratamento. Para as propriedades de módulo de ruptura (MOR) e módulo de elasticidade (MOE) à flexão estática foi empregada a norma DIN-52362 (1982), sendo utilizados 6 corpos de prova por tratamento. A resistência à tração perpendicular e a resistência ao arrancamento de parafuso foram avaliadas de acordo com as diretrizes da norma NBR 14810-2 (ABNT 2018), sendo utilizados 5 corpos de prova para cada ensaio. A densidade aparente dos painéis foi determinada com a média da densidade de cada um dos corpos de prova utilizados na avaliação das propriedades físicas e mecânicas.

Para uma melhor visualização da adesão ocorrida nos painéis, foram realizadas imagens com zoom de $0,75 \mathrm{x}$ através do microscópio estereoscópio SMZ 1500 (Nikon).

\section{Análise estatística dos dados}

Os dados foram analisados considerando um delineamento inteiramente casualizado, sendo submetidos à análise de variância (ANOVA) e teste Tukey, ambos a 5\% de significância. Os dados foram processados no software Sisvar

\section{Resultados e discussão \\ Propriedades físicas dos painéis}

Os valores obtidos para densidade aparente para os painéis produzidos não diferiram estatisticamente pelo teste de média Scott-Knott a $5 \%$ de significância (Tabela 1). De acordo com a NBR 14810-2 (ABNT 2018), os painéis podem ser 
classificados como de média densidade, pois apresentam densidade aparente superior a 0,551 g.cm-3. Observa-se que à medida que se aumenta o teor de adesivo nos painéis, os mesmos mantiveram seus valores médios para densidade aparente.

Tabela 1. Valores médios de densidade aparente, razão de compactação e umidade para os painéis produzidos.

\begin{tabular}{cccc} 
Adesivo (\%) & Densidade aparente $\left(\mathbf{g . c m} \mathbf{c m}^{-3}\right)$ & Razão de compactação & Umidade (\%) \\
\hline $6 \%$ & $0,618 \pm 0,05 \mathrm{~A}$ & $1,228 \pm 0,07 \mathrm{~A}$ & $8,93 \pm 0,22 \mathrm{~A}$ \\
$9 \%$ & $0,629 \pm 0,08 \mathrm{~A}$ & $1,250 \pm 0,05 \mathrm{~A}$ & $9,60 \pm 0,47 \mathrm{~A}$ \\
$12 \%$ & $0,624 \pm 0,04 \mathrm{~A}$ & $1,240 \pm 0,04 \mathrm{~A}$ & $9,50 \pm 0,29 \mathrm{~A}$ \\
\hline
\end{tabular}

Valores seguidos pela mesma letra na mesma coluna não diferem entre si pelo teste Tukey a 5\% de significância.

Comportamento semelhante foi observado por Brito et al. (2006), cujos autores observaram densidade aparente dos painéis produzidos com dois teores de ureia-formaldeído e maravalhas de Pinus elliottii Engelm. de 0,640 e 0,630 g.cm-3, para 6 e $8 \%$ de adesivo, respectivamente, não apresentando diferenças estatísticas significativas entre os tratamentos. Salienta-se que a densidade do painel está relacionada com a taxa de compactação das partículas, dependendo, também, da umidade do colchão e de outros fatores (Cunha et al. 2014).

Para a razão de compactação, os tratamentos não apresentaram diferenças estatísticas à medida que se elevou os teores de fenol-formaldeído nos painéis, seguindo o mesmo comportamento da densidade aparente. Os valores médios de razão de compactação variaram de 1,228 a 1,240 para painéis produzidos com 6 e $12 \%$ de adesivo fenolformaldeído, respectivamente. Resultados similares foram reportados por Guimarães Junior et al. (2016), Soares et al. (2017) e Andrade et al. (2018). Nenhum dos painéis produzidos ficaram dentro da faixa de 1,3 a 1,6, considerada como ideal por Maloney (1993) e Moslemi (1974) para que ocorra um contato adequado entre partículas de madeira e formação da ligação adesiva entre elas. Essa propriedade é um parâmetro de grande importância na resistência mecânica de painéis particulados, uma vez que painéis com maior razão de compactação provavelmente ocasionarão propriedades mecânicas superiores (Andrade et al. 2018).

A umidade variou de 8,93 a $9,60 \%$ para os painéis produzidos com diferentes teores de adesivo, abaixo do teor de umidade da câmara de climatização, que é de $12 \%$. Segundo Silva et al.
(2006), os teores de umidade obtidos neste trabalho estão na faixa para produtos oriundos de madeira reconstituída, inferiores ao da madeira sólida quando expostos em condições de temperatura e umidade semelhante. Ainda segundo os autores, este comportamento se deve por diferentes níveis de higroscopicidade, causada pela redução da madeira em partículas e posterior união com adesivo. Outro aspecto que contribui para a redução da higroscopicidade é a utilização de altas temperaturas e pressão na consolidação final do painel, sendo responsáveis pela degradação parcial das polioses, em particular dos grupos hidroxílicos, que atribuem caráter hidrofílico à madeira.

A norma brasileira NBR 14810-2 (Abnt 2018) estipula valores de umidade entre 5 e $11 \%$, sendo assim, todos os tratamentos se enquadram na norma citada.

\section{Absorção de água (AA) e Inchamento em espessura (IE) dos painéis \\ Foi observada diferença estatística significativa entre os tratamentos para as propriedades avaliadas. À medida que se aumentou os teores de adesivo nos painéis, estes apresentaram menores valores para AA após 2 e 24h (Tabela 2). Houve uma redução de $30,03 \%$ de absorção de água após $2 \mathrm{~h}$ de imersão e de $30,32 \%$ para absorção de água após $24 \mathrm{~h}$ de imersão para os painéis produzidos com $12 \%$ de adesivo. Esse comportamento pode ser explicado a uma maior distribuição de adesivo nas partículas dos painéis (Figura 3), resultando em painéis com menores porosidades.}

Tabela 2. Valores médios de absorção de água após 2 e 24h de imersão dos painéis aglomerados produzidos.

Adesivo (\%) Absorção de água 2h (\%) Absorção de água 24h (\%)

\begin{tabular}{ccc}
\hline $6 \%$ & $104,26 \pm 9,37 \mathrm{C}$ & $109,58 \pm 28,58 \mathrm{C}$ \\
$9 \%$ & $86,99 \pm 5,79 \mathrm{~B}$ & $97,47 \pm 5,90 \mathrm{~B}$ \\
$12 \%$ & $72,95 \pm 9,86 \mathrm{~A}$ & $81,79 \pm 10,32 \mathrm{~A}$ \\
\hline ela mesma letra na mesma coluna não diferem entre si pelo teste Tukey a $5 \%$ de significância.
\end{tabular}



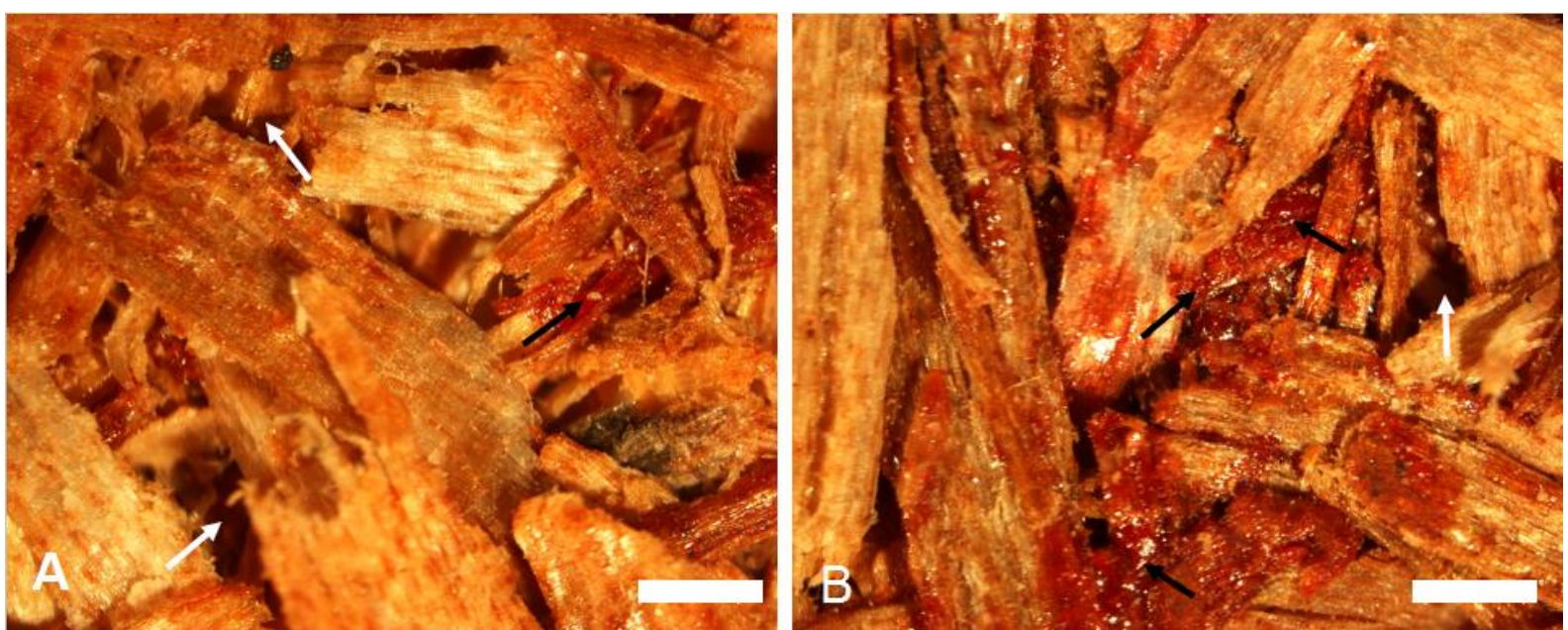

Figura 3. Painel aglomerado produzido com adesivo fenol-formaldeído. A) Painel aglomerado produzido com $6 \%$ de adesivo; B) Painel aglomerado produzido com 12\% de adesivo. Espaços vazios (seta branca), adesivo (seta preta). Barra de escala: $A-B=500 \mu \mathrm{m}$.

A diminuição da absorção de água conforme se aumentou o teor de adesivo está relacionada a um maior recobrimento das partículas, fazendo com que ocasione uma melhor ligação das mesmas e também proporcione menor disponibilidade dos sítios de sorção para a ligação com as moléculas de água, tendo por consequência a diminuição dos valores médios desta propriedade (Mendes et al. 2012).

A mesma tendência de decréscimo dos valores de absorção de água após 2 e $24 \mathrm{~h}$ foram observados por Rios et al. (2016), em que os autores trabalhando com diferentes teores de adesivo ureiaformaldeído notaram redução para ambas propriedades. Esse mesmo comportamento também foi observado por Dacosta et al. (2006), em que obtiveram uma redução de 36,00 e de $44,67 \%$ para absorção de água após 2 e 24 h de imersão em água, para painéis aglomerados confeccionados com maravalhas de Pinus elliotii Engelm. e teores de 4, 8 e $12 \%$ de adesivo ureia-formaldeído. Neste sentido, o comportamento dos painéis de maravalhas de
Eucalyptus grandis mostraram-se satisfatórios em comparação aos resultados encontrados na literatura.

Para IE após 2 e $24 \mathrm{~h}$ de imersão em água houve o mesmo comportamento observado para AA após 2 e 24h de imersão em água (Tabela 3). À medida que os teores de adesivo foram elevados, os painéis apresentaram uma melhor estabilidade dimensional, sofrendo menos inchamento. O aumento dos teores de adesivo fizeram com que os painéis apresentassem uma menor porosidade ocasionada pela maior quantidade de adesivo aderida às partículas (Figura 3). Esse comportamento de redução dos teores de inchamento em espessura também foram observados por Pedrazzi et al. (2006), relatando redução de 45,26 para $18,90 \%$ para inchamento em espessura após $24 \mathrm{~h}$ de imersão em água para painéis aglomerados produzidos com resíduos industriais celulósicos. Neste sentido, observa-se que os painéis aglomerados produzidos com maravalhas encontram-se abaixo daqueles verificados na literatura.

Tabela 3. Valores médios de inchamento em espessura após 2 e $24 \mathrm{~h}$ de imersão dos painéis aglomerados produzidos.

\section{Adesivo (\%) Inchamento em espessura 2h (\%) Inchamento em espessura 24h (\%)}

\begin{tabular}{ccc}
\hline $6 \%$ & $20,05 \pm 2,13 \mathrm{C}$ & $22,75 \pm 1,67 \mathrm{C}$ \\
$9 \%$ & $11,44 \pm 0,70 \mathrm{~B}$ & $13,09 \pm 0,89 \mathrm{~B}$ \\
$12 \%$ & $6,43 \pm 1,09 \mathrm{~A}$ & $8,08 \pm 1,04 \mathrm{~A}$ \\
\hline
\end{tabular}

Valores seguidos pela mesma letra na mesma coluna não diferem entre si pelo teste Tukey a 5\% de significância.

Comparando os resultados obtidos nesta pesquisa com as propriedades mínimas exigidas para painéis aglomerados de média densidade segundo a norma de comercialização CS 236-66 (1968), todos os painéis atenderam ao valor máximo para IE após 24 de imersão em água de $35 \%$. Já em relação à norma EN 312 (En 2003) para inchamento em espessura após $24 \mathrm{~h}$ de imersão em água, apenas os tratamentos contendo 9 e $12 \%$ de adesivo atenderam ao requisito de $14 \%$. Cabe ressaltar que um menor teor de adesivo utilizado nos painéis irá provocar uma redução nos custos finais dos painéis 
produzidos, uma vez que o adesivo pode chegar a representar 50\% do custo total de produção (Rios et al. 2016). Além disso, a utilização de menor teor de adesivo provocará uma menor emissão de formaldeído, substância que pode entrar no corpo humano e causar irritação dos olhos, nariz e garganta, lacrimação, tosse e náuseas, além de ser cancerígeno de acordo com a Organização Mundial da Saúde/OMS (Belini et al. 2015).

\section{Caracterização mecânica dos painéis \\ Flexão estática e tração perpendicular}

Observa-se na Tabela 4 que houve diferença estatística significativa para MOR, MOE e TP, em que os maiores valores médios para MOR, MOE e TP foi aquele referente aos painéis produzidos com $12 \%$ de adesivo. Os valores médios de MOR variaram de 3,27 MPa para painéis produzidos com $6 \%$ de adesivo fenol-formaldeído (T1) a 7,27 MPa para painéis produzidos com $12 \%$ de adesivo fenolformaldeído (T3). Esse comportamento pode ser explicado pelo fato de que quanto maior a quantidade de adesivo aplicada na produção dos painéis, melhor a ligação entre as partículas, o que resulta em maior resistência à flexão (Mendes et al. 2010).

Tabela 4. Valores médios de MOR, MOE e TP dos painéis aglomerados produzidos.

\begin{tabular}{cccc} 
Adesivo (\%) & MOR (MPa) & MOE (MPa) & TP (MPa) \\
\hline $6 \%$ & $3,27 \pm 0,83$ C & $385,83 \pm 104,66 \mathrm{C}$ & $0,13 \pm 0,10 \mathrm{~B}$ \\
$9 \%$ & $4,88 \pm 0,91 \mathrm{~B}$ & $637,53 \pm 117,09 \mathrm{~B}$ & $0,21 \pm 0,08 \mathrm{~B}$ \\
$12 \%$ & $7,27 \pm 0,99 \mathrm{~A}$ & $932,86 \pm 118,02 \mathrm{~A}$ & $0,38 \pm 0,07 \mathrm{~A}$ \\
\hline
\end{tabular}

Valores seguidos pela mesma letra na mesma coluna não diferem entre si pelo teste Tukey a 5\% de significância.

Rios et al. (2016) verificaram comportamento semelhante, uma vez que à medida que os autores elevaram os teores de adesivo nos painéis, os valores médios de MOR apresentaram crescimento. A mesma tendência foi observada na pesquisa de Pedrazzi et al. (2006), onde os autores observaram valores médios para MOR variando de 4,19 a 5,34 $\mathrm{MPa}$ para painéis aglomerados produzidos com $4 \mathrm{e}$ $12 \%$ de adesivo ureia-formaldeído.

Para MOE, os valores médios variaram de 385,83 a 932,86 MPa para painéis produzidos com 6 e $9 \%$ de adesivo fenol-formaldeído, respectivamente, sendo as médias estatisticamente diferentes.

Pedrazzi et al. (2006) também verificaram tendência de aumento para os valores médios de MOE ao passo que maiores quantidades de adesivo foram sendo adicionadas aos painéis. Os autores observaram um aumento de 720,89 para $994,69 \mathrm{MPa}$ para painéis aglomerados produzidos com 4 e $8 \%$ de adesivo ureia-formaldeído.

Da mesma forma, Mendes et al. (2010), Mendes et al. (2012) e Rios et al. (2016) observaram que, com o aumento do teor de adesivo nos painéis produzidos, os valores de MOR e MOE aumentam.

Nenhum dos tratamentos estudados atenderam ao requisito mínimo estabelecido pela norma ANSI 208.1 (1993) de $1700 \mathrm{MPa}$ para MOE e de $11 \mathrm{MPa}$ para MOR. Já em relação à norma de comercialização CS 236-66 (CS 1968), nenhum tratamento atendeu ao valor mínimo de $1716,16 \mathrm{MPa}$ para MOE e de 12,35 MPa para painéis aglomerados de média densidade produzidos com adesivo fenolformaldeído.
Apesar de nenhum tratamento atender às normativas supracitadas, os valores médios deste trabalho encontram-se próximos aos observados na literatura.

Observa-se no ensaio de tração perpendicular que os maiores valores foram conseguidos com os painéis produzidos com $12 \%$ de adesivo, e os mais baixos, com aqueles produzidos com teor de adesivo de 6 e $9 \%$. O aumento dos valores médios de tração perpendicular pode ser atribuído à maior disponibilidade de adesivo por unidade de área das partículas (Santos et al. 2009).

Segundo os autores Pedrazzi et al. (2006), Mendes et al. (2010) e Mendes et al. (2012) que trabalharam com painéis aglomerados e diferentes teores de adesivo observaram maiores valores de tração perpendicular quando da utilização de maiores teores de adesivo, independentemente do tipo de adesivo empregado na confecção dos painéis.

Os painéis produzidos com $12 \%$ de adesivo ureia-formaldeído apresentaram um ganho de $65,78 \%$ de resistência à tração perpendicular em relação àqueles produzidos com $6 \%$ do mesmo adesivo. Essa tendência também foi observada por Mendes et al. (2012), em que os autores notaram um aumento de 0,55 MPa para 0,84 MPa para painéis aglomerados de média densidade produzidos com adesivo ureia-formaldeído e combinação de partículas de bagaço de cana-de-açúcar e madeira de Pinus spp.

De acordo com a norma EN 312 (En 2003), apenas o tratamento composto por teor de $12 \%$ de adesivo atendeu ao requisito mínimo de 0,35 $\mathrm{MPa}$. Já em relação à norma de comercialização CS 23666 (Cs 1968), nenhum dos tratamentos avaliados 
atenderam ao requisito mínimo de $0,48 \mathrm{MPa}$ de resistência à tração perpendicular para painéis aglomerados de média densidade.

\section{Arrancamento de Parafuso}

Os resultados do ensaio de resistência ao arrancamento de parafuso indicaram que houve diferença estatística significativa entre os tratamentos nas duas condições avaliadas: face e topo (Tabela 5). Os resultados obtidos para os três tratamentos avaliados encontram-se próximos aos de
Oliveira et al. (2016) para as duas condições de ensaio. Os autores obtiveram valores médios para arrancamento de parafuso na condição topo de $474,64,710,0$ e de $520,73 \mathrm{~N}$ para painéis aglomerados de Pinus, Eucalyptus e bagaço de canade-açúcar, respectivamente. Já para a condição face, os autores encontraram os seguintes valores médios: $438,36,831,60$ e $582,52 \mathrm{~N}$, para os painéis produzidos com Pinus, Eucalyptus e bagaço de canade-açúcar, respectivamente.

Tabela 5. Valores médios de arrancamento de parafuso para as condições face e topo dos painéis aglomerados produzidos.

Adesivo (\%) Arrancamento de parafuso - Face (N) Arrancamento de parafuso - Topo (N)

\begin{tabular}{ccc}
\hline $6 \%$ & $414,36 \pm 57,98 \mathrm{~A}$ & $275,80 \pm 56,17 \mathrm{~A}$ \\
$9 \%$ & $739,70 \pm 206,40 \mathrm{~B}$ & $520,85 \pm 137,55 \mathrm{~B}$ \\
$12 \%$ & $743,00 \pm 125,42 \mathrm{~B}$ & $466,13 \pm 57,17 \mathrm{AB}$ \\
\hline
\end{tabular}

Valores seguidos pela mesma letra na mesma coluna não diferem entre si pelo teste Tukey a 5\% de significância.

Já em relação ao estudo realizado por Cunha et al. (2014), os valores médios encontrados neste estudo apresentam-se abaixo ao dos autores, que obtiveram resistência ao arrancamento de parafuso na condição de topo de $1163,93 \mathrm{~N}$ e de $1321,06 \mathrm{~N}$ para a condição de face.

Nenhum dos tratamentos estudados atenderam aos requisitos mínimos exigidos pela norma NBR 14810-2 (ABNT 2018), que estabelece limites mínimos de $1020 \mathrm{~N}$ para arrancamento de superfície e $800 \mathrm{~N}$ para topo. O mesmo ocorreu em relação à norma americana ANSI A 208.1 (2009), que fixa $900 \mathrm{~N}$ para superfície e $800 \mathrm{~N}$ para topo independente do sentido de aplicação, onde nenhum dos painéis produzidos obedeceram tal normativa. Segundo Cunha et al. (2014), os baixos valores encontrados para essa propriedade podem ser atribuídos a baixa densidade dos painéis. Painéis aglomerados de alta densidade apresentam melhores valores de resistência ao arrancamento de parafuso que painéis de média e baixa densidade.

\section{Conclusões}

Não houve diferenças estatísticas para as propriedades físicas dos painéis aglomerados à medida que se elevou os teores de adesivo fenolformaldeído. A absorção de água e inchamento em espessura, ambos após 2 e 24 h, diminuíram com o aumento do teor de adesivo. Mesmo comportamento foi observado para os módulos de elasticidade e ruptura no ensaio de flexão estática e para tração perpendicular. Já para arrancamento de parafuso, os resultados aumentaram à medida que se elevou o teor de adesivo nos painéis.

\section{Referencias}

Amaral D, Zaú AS, Gama DC, Albuquerque E, Silva FJ (2018) Aproveitamento de resíduo madeireiro em um município amazônico. Biodiversidade, 17(2):2233.

American National Standard Institute (2009) Matformed wood particleboard: specification ANSI A 208.1. Leesburg: Composite Panel Association, 13 p.

American Society for Testing and Materials (2012) Annual book of ASTM: D-1037: Standard methods of evaluating properties of wood-base fiber and particles materials. Philladelphia, 31p.

Andrade LMF, Scatolino MV, Faria DL, César AA, Mendes LM, Guimarães Junior JB (2018) Inclusão do resíduo de polpação da celulose na produção de painéis aglomerados de média densidade. Scientia Forestalis, 46(120). doi: 10.18671/scifor.v46n120.11

Associação Brasileira de Normas Técnicas (2018) NBR 14810: Painéis de partículas de média densidade Parte 2: Requisitos e métodos de ensaio. $71 \mathrm{p}$.

Baldin T, Silveira AG, Vidrano BRA, Cancian LC, Spatt LL, Haselein CR (2016) Qualidade de aglomerados produzidos com diferentes proporções de madeira e capim-annoni. Revista Brasileira de Ciências Agrárias, 11(3):230-237. doi:10.5039/agraria.v11i3a5376

Belini UO, Fiorelli J, Savastano Junior H, Leite MK, Tomazello Filho M (2015) Formaldeído livre em painéis de eucalipto e cana-de-açúcar. Ciência da Madeira, 6(2):94-95. doi: 10.12953/21776830/rcm.v6n2p94-99 
Brito EO, Sampaio LC, Oliveira JN, Batista DC (2006) Chapas de madeira aglomerada utilizando partículas oriundas de madeira maciça e de maravalhas. Scientia Forestalis, (72):17-21.

Cabral MR, Fiorelli J, Cravo JCM, Savastano H (2017) Painéis de partículas com maravalha de Pinus spp. e fibra de sisal. Scientia Forestalis, 45(114):353362. doi: 10.18671/scifor.v45n114.11

Commercial Standard (1968) CS 236-66: mat formed wood particleboard. [S.1.].

Cunha AB, Longo BL, Rodrigues AA, Brehmer DR (2014) Produção de painéis de madeira aglomerada de Eucalyptus benthamii, Eucalyptus dunnii e Eucalyptus grandis. Scientia Forestalis, 42(102):259-267.

Dacosta LPE, Haselein CR, Santini EJ, Schneider PR, Calegari L (2006) Propriedades físicas de chapas de partículas aglomeradas fabricadas com resíduos do processamento mecânico da madeira de Pinus elliotii Engelm. Ciência Florestal, 15(4):421-429. doi: 10.5902/198050981879

Deutsches Institut fur Normung (1982) DIN 52362: Testing of wood chipboards, bending test, determination of bending strength. Berlin, $40 \mathrm{p}$.

European Standard (2003) EN 312: particleboards: specifications. British Standard Institution, London, $22 \mathrm{p}$.

Fagundes HAV (2003) Produção de madeira serrada e geração de resíduos do processamento de madeira de florestas plantadas no Rio Grande do Sul. Dissertação (Mestrado em Engenharia Civil), Universidade Federal do Rio Grande do Sul. 173p.

Fiorelli J, Ramos RD, Sayama JT, Barrero NMG, Pallone EMJA (2014) Particleboards with waste wood from reforestation. Acta Scientiarum. Technology, 36(2):251-256. doi: 10.4025/actascitechnol.v36i2.18757

Guimarães Junior JB, Xavier MM, Santos TS, Protásio TP, Mendes RF, Mendes LM (2016) Inclusão de resíduo da cultura de sorgo em painéis aglomerados de eucalipto. Pesquisa Florestal Brasileira, 36(88):435- 442. doi: 10.4336/2016.pfb.36.88.1036

Hazrati-Behnagh M, Zarea-Hosseinabadi H, DaliriSosefi M, Abginehchi Z, Hemmati A (2016) Mechanical and insulating performances of ultralight thick particleboard from sugarcane residues and woods planer shaving. European Journal of Wood and Wood Products, 74(2):161168. doi: 10.1007/s00107-015-0989-7

Indústria Brasileira de Árvores - IBÁ. Anuário 2019: ano base 2019. São Paulo: Ibá, 2019. 80 p.

Iwakiri S, Vianez BF, Weber C, Trianoski R, Almeida VC (2012) Avaliação das propriedades de painéis aglomerados produzidos com resíduos de serrarias de nove espécies de madeiras tropicais da Amazônia. Acta Amazonica, 42(1):59-64. doi: 10.1590/S0044-59672012000100007

Maloney TM (1993) Modern particleboard \& dryprocess fiberboard manufacturing. Backbeat.

Mendes RF, Mendes LM, Guimarães Junior JB, Santos RC, César AAS (2012) Efeito da associação de bagaço de cana, do tipo e do teor de adesivo na 
produção de painéis aglomerados. Ciência Florestal, 22(1):161-170. doi: 10.5902/198050985088

Mendes RF, Mendes LM, Abranches RAS, Santos RC, Guimarães Junior JB (2010) Painéis aglomerados produzidos com bagaço de cana e madeira de Eucalipto. Scientia Forestalis, 38(86):285-295.

Moslemi AA (1974) Particleboard. Illinois: Southern Illinois University Press. 244p.

Oliveira SL, Mendes RF, Mendes LM, Freire TP (2016) Particleboard panels made from sugarcane bagasse: characterization for use in the furniture industry. Materials Research, 19(4):914-922. doi: 10.1590/1980-5373-MR-2015-0211

Pedrazzi C, Haselein CR, Santini EJ, Schneider PR (2006) Qualidade de chapas de partículas de madeira aglomerada fabricadas com resíduos de uma indústria de celulose. Ciência Florestal, 16(2):201212. doi: 10.5902/198050981899

Rios PD, Pereira GF, Vieira HC, Grubert W, Cunha AB, Brand MA (2016) Avaliação do potencial da madeira de Pinus patula Schltdl. \& Cham para a produção de painéis de madeira aglomerada. Scientia Forestalis, 44(110):497-508. doi: $10.18671 /$ scifor.v44n 110.22

Santos RC, Mendes LM, Mori FA, Mendes RF (2009) Chapas de partículas aglomeradas a partir de resíduos gerados após a extração de óleo da madeira de candeia (Eremanthus erytropapus). Scientia Forestalis, 37(84):437-446.

Silva CP, Vieira RS, Silva IC, Dornelas ASP, Baraúna EP (2017) Quantificação de resíduos produzidos nas indústrias madeireiras de Gurupi, TO. Floresta e Ambiente, 24: e00065613. doi: $10.1590 / 2179-8087.065613$

Silva GA, Mendes LM, Calegário N, Mori FA, Silva JRM, Santos IF (2006) Estimativa de umidade de equilíbrio de painéis de madeira. Scientia Forestalis, (70):23-29.

Silva VU (2019) Viabilidade técnica do reaproveitamento da maravalha de forma integral na produção de painéis de partículas. Tese, Escola de Engenharia de São Carlos, Universidade de São Paulo. 98p.

Soares SS, Guimarães Junior JB, Mendes LM, Mendes RF, Protásio TP, Lisboa FJN (2017) Valorização do bagaço de cana-de-açúcar na produção de painéis aglomerados de baixa densidade. Ciência da Madeira, 8(2):64-73. doi: 10.12953/2177-6830/rcm.v8n2p64-73 